\title{
A Study of Dynamics to Solve The Human Initial Gesture Based on the Known Information of the Man-Rifle System
}

\author{
ZHANG ben-jun ${ }^{1, a}$, LIU chuan ${ }^{1, a}$, LIU guangsheng ${ }^{1, a}$, JIA changzhi, ${ }^{1, a}$ \\ ${ }^{1}$ Department of Guns Engineering, Ordnance Engineering College, Shijiazhuang, 050003; \\ a E-mail: Iczchuan@163.com
}

Keywords: man-rifle system, initial gesture, part information, dynamics

\begin{abstract}
A method which uses the dynamical model is raised to solve the human initial gesture of the man-rifle system. It uses the known information of part of limbs to build the truthfulness constraint, and then solve the problem with the dynamics of rigid multi-body. Using this method and combined using ADAMS and LifeMod to calculate the human initial gesture of prone position loaded and prone position fire of certain universal stutterer, the result is truthfulness and by which proves the feasibility of this method, it provides a new method to solve the human initial gesture of the man-rifle system, meanwhile, it also provides a new thinking to solve the same kind problems.
\end{abstract}

\section{Introduction}

The operating gesture is the decisive factor to assess whether the design position of the parts are suitable when the shooter hold a gun. It is a fundamental work to locate human joint when building the model of the man-rifle system, and it is also the precondition to make sure that the built model can reflect the interaction of the human and gun actually when shooting. Generally [1,2], direct measurement method is used to confirm the human gesture in the man-rifle system, which advantage is the acquired result is completely genuine and believable, but it also has apparent disadvantages. Direct measurement method requires actual human and gun, while these two conditions may not exist accurately which limits the use of the direct measurement method.

In recent years, multi-body dynamic models are used widely in the solve of the human motion. Basing the built rigid multi-body dynamics models and other theories, Ayoub, Xu Guoyu, Blajera , Ohta, Yang Yiyong analyzed the problems they studied [3-7]. While their studies focus on the movement of human, they had no idea to confirm the human initial gesture.

To the man-rifle system, a part of limbs location is known when the shooter operates the gun. This paper intends to use these known information, and using multi-body dynamics theory to solve the overall gesture of the human initial gesture of the man-rifle system, intending to get a method to solve the overall gesture of the human initial gesture.

\section{The building of the human model}

\section{A. Determining the parameter of the human model}

According to characteristic of human body, this paper divides the human model into sixteen rigid parts, include head, top body, middle body, below body, left and right big arm, left and right forearm, left and right hand, left and right thigh ham, left and right shank, left and right foot.

Establishing the coordinate system, the $\mathrm{Y}$ axis is vertical axis, the front of the sagittal axis is $\mathrm{Z}$ axis, and the $\mathrm{X}$ axis is cross axis, the positive direction is confirmed by right-hand rule. The direction of local coordinate system of every rigid bodies are same with overall coordinate system before calculating. This paper uses the software Lifemod of the company Research Group to confirm the geometric properties and quality attributes of every body parts. The calculation correlation of the software is:

$$
\begin{aligned}
L_{i} & =L_{i}(G, H, W, A) \\
M_{i} & =M_{i}(G, H, W, A)
\end{aligned}
$$


Where, $L_{i}$ is the geometric properties vector of the $i$ part, $M_{i}$ is the quality attributes vector, $G$ is sex, $H$ is height, $W$ is weight, $A$ is age. The shooter in this paper is a man, his height is $1800 \mathrm{~mm}$, his weight is 77 kilograms and he is 21.

\section{B. The processing of the joint}

In the real human body, every part of the body is jointed by joint, these parts which jointed by joints can only turn, they can not slide. Therefore, these joints are looked as ball joint when the model is built. To make sure the stability of the calculate and the limit angle of joints, add internal moment like (3) to every joints:

$$
\left\{\begin{array}{cc}
K_{m} \alpha_{m}+C_{m} \alpha_{m} & \alpha_{L m} \leq \alpha_{m} \leq \alpha_{U m} \\
K_{\lim } \alpha_{m}+C_{m} \alpha_{m} & \alpha_{m}<\alpha_{L m} \text { 或 } \alpha_{m}>\alpha_{U m}
\end{array}\right.
$$

Where, $m$ is the $m$ joint, $\alpha_{m}$ is three-dimensional column, its elements represent the turn angle of the vertical plane, level, and coronal plane, $\alpha_{L m}$ and $\alpha_{U m}$ are three-dimensional column too, they represent the upper limit and the floor limit of every joint, these limit angle can find in the literature [8], $K_{m}$ and are all three order diagonal matrix, they represent stiffness matrix and damping matrix. $K_{\text {lim }}$ is three order diagonal matrix, it represents the stiffness when the turning angle of the joint exceed the limited angle, its elements are one hundred times of the max elements of stiffness matrixes.

\section{The building of the dynamics equation}

\section{A. Using of the known information}

Choosing the centroid Cartesian coordinate system and Euler Angles as the generalized coordinate system:

$$
q_{i}=[x, y, z, \psi, \varphi, \theta]_{i}^{T}
$$

To man-rifle system, the location and direction of the contact point of the hands and bodies with the gun are confirmed, which gives the border terms of the built rigid multi-body model, it means that the $q_{a}\left(t_{0}\right)$ is known. The $a$ in the equation means the a rigid body which can be confirmed its location and direction, $t_{0}$ is the end time of the dynamic calculation. Adding holonomic constraints to these rigid bodies which location are confirmed:

$$
f_{a}\left(q_{a}, t\right)=0
$$

This constraints must meet requirements of $q_{a}\left(t_{0}\right)$. Actually, the addition of these constraints are equal to adding prime mover to the human model, it confirms the movement locus of the rigid bodies which final location are confirmed. The final result must be one of the actual situations, due to the constrain is meet the requirements of $q_{a}\left(t_{0}\right)$. But it is related to these movements to find which actual situation is same as the result. These movements can be smooth curves, they can also be piecewise curves, but the process they confirm need to be same as the actually process, it will be more accurate if it is closer to the actually process.

\section{B. Establishing and solve the kinetic equation}

Using the Lagrange equation with a multiplier to give the kinetic equations and constraint equations of the human model like equation (6) and equation (7):

$$
\frac{d}{d t}\left(\frac{\partial T}{\partial \dot{q}}\right)^{T}-\left(\frac{\partial T}{\partial q}\right)^{T}+\Phi_{q}^{T} \rho+\psi_{\dot{q}}^{T} u=Q
$$




$$
\left\{\begin{array}{l}
\Phi(q, t)=0 \\
\psi(q, \dot{q}, t)=0
\end{array}\right.
$$

Where, $T$ is the kinetic energy of the model, $\Phi$ is the holonomic constraint equations of the model, $\psi$ is the non-holonomic constraint equations of the model, $\rho$ and $u$ are the Lagrange equation multipliers, $Q$ is generalized column matrix. For these equations, we can solve them with ADAMS [9], the process is as follows:

Let $u=d q / d t$ and the two equations aforesaid can be write as follows:

$$
\left\{\begin{array}{c}
F(q, u, \dot{u}, \lambda, t)=0 \\
G(u, \dot{q})=u-\dot{q}=0 \\
\Phi(q, t)=0
\end{array}\right.
$$

Where, $F$ is the differential equation of the dynamics system, $\lambda$ is constraint reaction and force array. let $y=\left[q^{T}, u^{T}, \lambda^{T}\right]^{T}$ as the state vector of the system, and then we can use the state vector of current time and Taylor series to predict the system's vector of next time, it is:

$$
y_{n+1}=y_{n}+\frac{\partial y_{n}}{\partial t} h+\frac{1}{2 !} \frac{\partial^{2} y_{n}}{\partial t^{2}} h^{2}+\ldots
$$

Where, $h=t_{n+1}-t_{n}$ is time step. Generally the state vector we get needs to be revised, and then use Gear ${ }^{k+1}$ dielectric integral solving and revising :

$$
y_{n+1}=-h \beta_{0} \dot{y}_{n+1}+\sum_{i-1}^{k} \alpha_{i} y_{n-i+1}
$$

Where, $\beta_{0}$ and $\alpha_{i}$ are the coefficient of the Gear program. Unfold (7) at $t=t_{n+1}$ :

$$
\left\{\begin{array}{c}
F\left(q_{n+1}, u_{n+1}, u_{n+1}, \lambda_{n+1}, t_{n+1}\right)=0 \\
G\left(u_{n+1}, q_{n+1}\right)=u_{n+1}-\left(\frac{-1}{h \beta_{0}}\right)\left(q_{n+1}-\sum_{i=1}^{k} \alpha_{i} q_{n-i+1}\right)=0 \\
\Phi\left(q_{n+1}, t_{n+1}\right)=0
\end{array}\right.
$$

Re-corrected Newton-Raphson program is used to solved (11), get the iterative formula as (12):

$$
\left\{\begin{array}{c}
F_{j}+\frac{\partial F}{\partial q} \Delta q_{j}+\frac{\partial F}{\partial u} \Delta u_{j}+\frac{\partial F}{\partial \dot{u}_{j}} \Delta u_{j}+\frac{\partial F}{\partial \lambda} \Delta \lambda_{j}=0 \\
G_{j}+\frac{\partial G}{\partial q} \Delta q_{j}+\frac{\partial G}{\partial u} \Delta u_{j}=0 \\
\Phi_{j}+\frac{\partial \Phi}{\partial q} \Delta q_{j}=0
\end{array}\right.
$$

Where, $j$ is the $j$ time iterative, $\Delta q_{j}=q_{j+1}-q_{j}, \Delta u_{j}=u_{j+1}-u_{j}, \Delta \lambda_{j}=\lambda_{j+1}-\lambda_{j}$. Basing expression (10) and expression (11) we can get:

$$
\Delta u_{j}=-\left(\frac{1}{h \beta_{0}}\right) \Delta u_{j}, \frac{\partial G}{\partial q}=\left(\frac{1}{h \beta_{0}}\right) I, \frac{\partial G}{\partial u}=I
$$

Where, $I$ is unit matrix, introduce expression (13) into expression (12) and then get the iteration matrices: 


$$
\left(\begin{array}{ccc}
\frac{\partial F}{\partial q} & \left(\frac{d F}{\partial u}-\frac{1}{h \beta_{0}} \frac{\partial F}{\partial \dot{u}}\right) & \frac{d F}{\partial \lambda} \\
\left(\frac{1}{h \beta_{0}}\right) I & I & 0 \\
\frac{d \Phi}{\partial q} & 0 & 0
\end{array}\right)\left\{\begin{array}{l}
\Delta q \\
\Delta u \\
\Delta \lambda
\end{array}\right\}_{j}=\left\{\begin{array}{l}
-F \\
-G \\
-\Phi
\end{array}\right\}_{j}
$$

The solution meet error will be get after constant iterative.

\section{Calculation example}

A typical fault of certain stutterer is it is hard to start seating, while it is need to solve the boundary condition during the process of start seating if we want to study the problem of start seating, which requires to confirm the human initial gesture. It's need to confirm the human initial gesture during shooting either if studying the human body's influence on shooting precision during shooting and studying the stutterer's influence on human body. This paper takes these two initial gesture for example, using the method mentioned before, combining LifeMod and ADAMS to solve the problem.

During the process of prone position loaded and prone position fire, the body and the direction of fire should collinear, which makes each joint of body can only rotate in the vertical plane. Joints of body and joints of left arm are same as the joints of neck during prone position loaded and prone position fire, there are only the joints of right arm different with them, so, only the gesture of right arm during prone position fire has been solved after solving the initial gesture of prone position loaded.

Table. 1 Each joint's angle during prone position loaded

\begin{tabular}{cccc}
\hline & vertical plane & Horizontal plane & Coronal plane \\
\hline Joint of head & -35.45 & 11.33 & 30.12 \\
Joint of chest & -8.49 & 0 & 0 \\
Joint of waist & -14 & 0 & 0 \\
Joint of left shoulder & -125.15 & 22.1 & -11.57 \\
Joint of left elbow & 0 & -8.62 & -95.53 \\
Joint of left wrist & -6.66 & 0 & -12.55 \\
Joint of right shoulder & -116.65 & 20.7 & -30.69 \\
Joint of right elbow & -77.92 & 49.06 & 0 \\
Joint of right wrist & 2.15 & 0 & -5.37 \\
\hline
\end{tabular}

Set the firing line of this stutterer at $310 \mathrm{~mm}$, and the locations of the stutterer's buttstock, grip, bolt handle and aiming point will be confirmed. Establishing the holonomic constraints at shoulders, hands and head with the mentioned method and then the initial gesture in the two situations can be solved. During the solution procedure, the first step is to solve the gesture of body and then basing the gesture, the gestures of left arm and right arm and head can be solved, the locus curves of left arm and right arm are all constitute with three parts. The results are presented at table 2, table 3 and picture 1 and picture 2:

Table. 2 Joint of right arm during prone position fire

\begin{tabular}{cccc}
\hline & vertical plane & Horizontal plane & Coronal plane \\
\hline Joint of shoulder & -97.43 & 7.25 & -8.55 \\
Joint of elbow & -106.58 & -15.34 & 4.45 \\
Joint of wrist & 30.56 & 0 & 5.82 \\
\hline
\end{tabular}




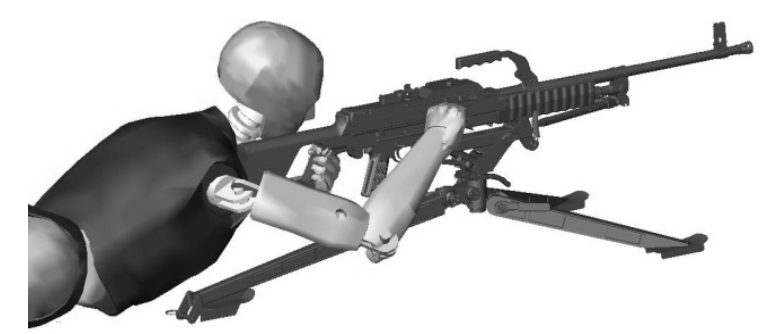

Fig. 1 Plot of human initial gesture during prone position loaded

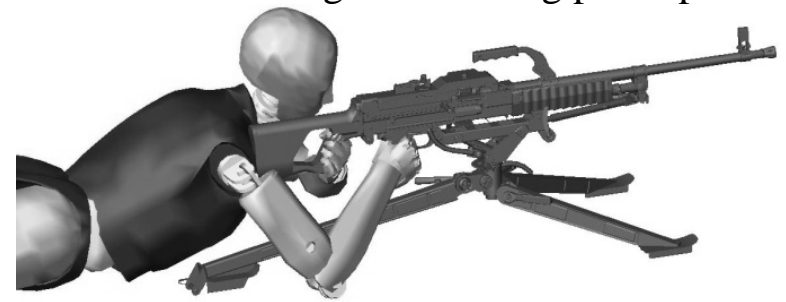

Fig. 2 Plot of human initial gesture during prone position fire

We can get the information from these pictures and tables that the results we get are consistent with the two actual situations, from which proves the feasibility of the method.

\section{Conclusions}

A method which uses the dynamical model is raised in this paper to solve the human initial gesture of the man-rifle system. It uses the known information of part of limbs to build the truthfulness constraint, and then solve the problem with the dynamics of rigid multi-body. Using this method and combinedusing ADAMS and LifeMod to calculate the human initial gesture of prone position loaded and prone position fire of certain universal stutterer, the result is truthfulness and by which proves the feasibility of this method, it provides a new method to solve the human initial gesture of the man-rifle system, meanwhile ,it also provides a new thinking to solve the same kind problems.

\section{REFERENCES}

[1] Chen Ming. Research of Rigid-Flexible Coupling Firing Dynamics of Machine Gun Considering of Stochastic Factors and Non-linear Factors[D]. Shijiazhuang: Ordnance Engineering College, 2009. (in Chinese)

[2] Young-Shin Lee, Young-Jin Choi, Kyu-Hyun Han, et al. A Study on the Human Impulse Characteristics Of the Standing Shooting Posture[J]. Key Engineering Materials, 2005, 297-300(4): 2314-2319. (in English)

[3] Ayoub M M. Simulation for sagittal plane lifting activities[J]. Occupational Ergonomics, 2002/2003:141-151.(in English)

[4] Xu Guoyu, Mei Xuesong Wu Xutang, The Study on the Model of the Upper Part of a Human Body[J]. Chinese Journal of Applied Mechanics, 2000, 17(1): 125-131. (in Chinese)

[5] Wojciech B., Czaplickib A.. Modeling and inverse simulation of somersaults on the trampoline [J]. Journal of Biomechanics, 2001,34(12):1619-1629. (in English)

[6] Ohta Ken, Svinin Mikhail M., Luo Zhiwei, Hosoe Shifeyuki. et al. Optimal trajectory formation of constrained human arm reaching movements[J]. Biological Cybernetics, 2004, 91(1): 23-36. (in English)

[7] Yang Yiyong, Wang Rencheng, Wang Yanli, Jin Dewen. Forward dynamic analysis of human lower limb neuron musculoskeletal system. J Tsinghua Univ(Sci \&Tech), 2006, 46(11): 1172-1175. (in Chinese)

[8] Mao Enrong, Zhang Hong, Song Zhenghe. Ergonomics of Vehicle [M]. Beijing: Beijing 
University of Science and Technology press, 2007. (in Chinese)

[9] Li Jun, Xing Junwen, Tan Wenjie. Example Tutorial of ADAMS [M]. Beijing: Beijing University of Science and Technology press, 2002. (in Chinese) 